\title{
Structural alterations of foreskin caused by chronic smoking may explain high levels of urethral reconstruction failure using foreskin flaps
}

\author{
João P. Rosado, Luciano A. Favorito, Andre G. L. Cavalcanti, Waldemar S. Costa, Luiz E. M. Cardoso, \\ Francisco J. B. Sampaio
}

Urogenital Research Unit from State University from Rio de Janeiro, RJ, Brazil

\section{ABSTRACT}

Objectives: The aim of the present study was to perform a stereological and biochemical analysis of the foreskin of smoker subjects.

Materials and Methods: Foreskin samples were obtained from 20 young adults (mean = 27.2 years old) submitted to circumcision. Of the patients analyzed, one group $(\mathrm{n}=10)$ had previous history of chronic smoking (a half pack to 3 packs per day for 3 to 13 years (mean $=5.8 \pm 3.2$ ). The control group included 10 nonsmoking patients. Masson's trichrome stain was used to quantify the foreskin vascular density. Weigert's resorcin-fucsin stain was used to assess the elastic system fibers and Picrosirius red stain was applied to study the collagen. Stereological analysis was performed using the Image J software to determine the volumetric densities. For biochemical analysis, the total collagen was determined as $\mu \mathrm{g}$ of hydroxyproline per mg of dry tissue. Means were compared using the unpaired t-test $(\mathrm{p}<0.05)$.

Results: Elastic system fibers of smokers was $42.5 \%$ higher than in the control group (p $=0.002)$. In contrast, smooth muscle fibers $(p=0.42)$ and vascular density $(p=0.16)$ did not show any significant variation. Qualitative analysis using Picrosirius red stain with polarized light evidenced the presence of type I and III collagen in the foreskin tissue, without significant difference between the groups. Total collagen concentration also did not differ significantly between smokers and non-smokers $(73.1 \mu \mathrm{g} / \mathrm{mg} \pm 8.0 \mathrm{vs} .69 .2 \mu \mathrm{g} /$ $\mathrm{mg} \pm 5.9$, respectively, $\mathrm{p}=0.23$ )

Conclusions: The foreskin tissue of smoking patients had a significant increase of elastic system fibers. Elastic fibers play an important role in this tissue's turnover and this high concentration in smokers possibly causes high extensibility of the foreskin. The structural alterations in smokers' foreskins could possibly explain the poor results in smoking patients submitted to foreskin fasciocutaneous flaps in urethral reconstruction surgery.
ARTICLE INFO

\section{Key words:}

Foreskin; Smoking;

Urethra; Histology

Int Braz J Urol. 2012; 38: 529-35

Submitted for publication: January 01, 2012

Accepted after revision: March 28, 2012

\section{INTRODUCTION}

Male urethral reconstruction is one of the oldest problems in reconstructive surgery and has always been a great challenge to urologists (1). Urethral stricture is a fibrotic process resulting in a scar tissue with low compliance, decreasing the caliber of the urethral lumen. Depending on the degree of stenosis, urethral stricture has variable impact on the patient's micturition (2).

There are wide spectrums of different pathologies that can cause reduction of the urethral 
caliber, like lichen sclerosis, hypospadias, trauma, infections and more recently iatrogenic injuries related to urological procedures $(3,4)$. The surgical techniques described to correct urethral stenosis in the past decade have allowed remarkable advances in the treatment of this condition. However, although there are now several options, no single one is completely secure and free of complications. Anastomotic urethroplasty with excision of scar tissue is associated with better long term results (5). When this procedure is impossible due to the large extension of the lesion, tissue transfer techniques can be chosen, like fasciocutaneous flaps or grafts (oral mucosa or genital skin) (6).

Wessels (7) in a meta-analysis studied the use of graft versus free flap and found no differences in the procedures for the treatment of bulbar strictures, but when dealing with long lesions of the urethra, or patients with an overly compromised layer, transverse distal penile island flap is the main form of treatment, requiring long follow-up. Transverse distal penile island flap has been used for one-stage urethral reconstruction and has led to good functional and cosmetic results. The penile skin associated with the tunica dartos is well-vascularized by superficial external pudendal vessels. It is hairless and the pedicle can reach anywhere from the external meatus to the prostatic urethra for reconstruction as a patch or tube (1).

It has been speculated in two reliable articles, one studying the long term efficacy of distal penile circular fasciocutaneous flaps and other a multivariate analysis of risk factors for longterm urethroplasty, that smokers might experience worse results after urethral reconstruction. Nevertheless, no structural evidence has been reported to date $(8,9)$.

The aim of the present study was to perform a structural analysis of the foreskin in smokers.

\section{MATERIALS AND METHODS}

The present work received approval from the institutional review committee. This work was carried out in accordance to the ethical standards of the institutional committee on human experimentation.
Foreskin samples were obtained from 20 young adults, ranging in age from 23 to 36 years (mean $=27.2$ years old) submitted to circumcision from July 2008 to July 2010. All patients presented true phimosis with foreskin stenosis. All 20 patients presented total impossibility of foreskin retraction. The exclusion criteria were patients with clinical suspicion of balanitis xerotica obliterans.

After surgery, dorsal and ventral regions of the foreskin were carefully demarcated with a suture. Of the patients analyzed, one group ( $\mathrm{n}=$ 10) had previous history of chronic smoking of a half pack to 3 packs per day for 3 to 13 years (mean $=5.8 \pm 3.2$ years). The control group was composed of 10 non-smokers; those patients never smoked and didn't not cohabite with a smoker.

The foreskin tissue samples was separated and fixed in 10\% buffered formalin and routinely processed for paraffin embedding, and $5 \mu \mathrm{m}$ thick sections were obtained at $200 \mu \mathrm{m}$-intervals. Smooth muscle, connective tissue, elastic system fibers and collagen were studied by histochemical, immunohistochemical and biochemical methods.

Sections were stained with haematoxylin-eosin to assess the integrity of the tissue. We performed the following stainings: Masson's trichrome, in order to quantify the foreskin vascular density; Weigert Resorcin Fucsin with previous oxidation in order to observe elastic system fibers; and Picrosirius Red with polarization for observation of different collagen types. Connective tissue, smooth muscle and elastic system fibers were quantified by a stereological method (2).

From each foreskin fragment, ten different sections were selected. Five from the ventral area end five from dorsal area, and five fields of each section were selected. All selected fields were photographed with an Olympus DP70 camera coupled to an Olympus BX51 microscope. The images were processed using the Image Pro software and the fibers were quantified using the image $J$ software to determine the volumetric density ( $\mathrm{Vv}$ ) of each component (Figure-1).

The immunohistochemistry analysis of the elastic fibers used the anti-elastin method with positive and negative controls (ABCAM). The slides were previously treated with poly-L-lysine for better adherence of the sections. 
Figure 1 - Photomicrography showing the morphometric analysis of the foreskin. Quantification of vascular density of the foreskin in a 23 years old smoker using the image J Test Grid software. Masson's trichrome x200.

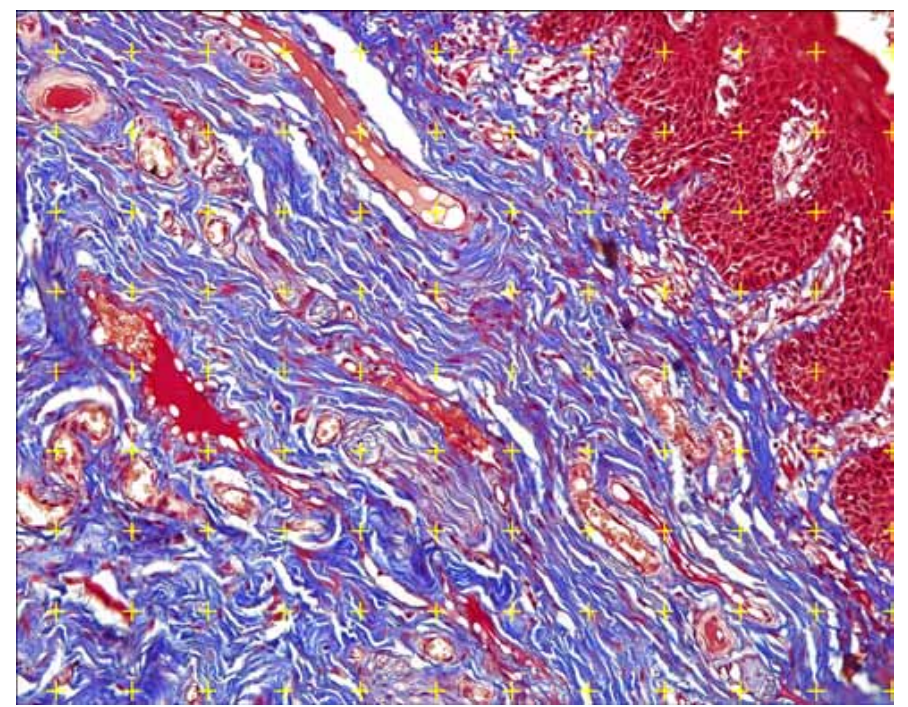

For the biochemical analysis of the collagen, tissue samples were fixed in acetone. The concentration of total collagen in the foreskin was determined by a colorimetric hydroxyproline assay. In this process, 5 to $14 \mathrm{mg}$ of dry, defatted bladder tissue was hydrolyzed in $6 \mathrm{~N} \mathrm{HCl}$ for 18 hours at $118^{\circ} \mathrm{C}$, as previously described (10). The assay was then carried out in the neutralized hydrolysate using the chloramine T method (11). The results were expressed as micrograms of hydroxyproline per milligram of dry, defatted tissue.

Means were statistically compared using the unpaired t- test $(p<0.05)$ with the Graph Pad Prism software.

\section{RESULTS}

The foreskin tissue was analyzed in two different regions, the ventral area and dorsal area. We did not find any statistical difference between the ventral and dorsal areas. In non-smokers the elastic fibers $(p=0.32)$, vascular density $(p=0.27)$ and smooth muscle fibers $(\mathrm{p}=0.46)$ did not show statistical difference between ventral and dorsal areas. With this statistical information, we chose not to maintain the differentiation between the dorsal and ventral area during the rest of the study.

The elastic system fibers in the foreskin of smokers was $42.5 \%$ higher than in the control group $(32.0 \pm 6.3$ vs. $45.6 \pm 10.1$, respectively; $p$ $=0.002$ ) (Figure-2). In contrast, the smooth muscle fibers $(\mathrm{p}=0.42)$ and the vascular density $(\mathrm{p}=$ 0.16 ) did not show any significant variation between smokers and non-smokers (Table-1).

The qualitative analysis using Picrosirius red stain with polarized light evidenced the presence of type I and III collagen in the foreskin with a predominance of type I collagen without a significant difference in its basic characteristics between the groups. The total collagen concentration also did not differ significantly between smokers and non-smokers $(73.1 \mu \mathrm{g} / \mathrm{mg} \pm 8.0 \mu \mathrm{g} / \mathrm{mg}$ vs. $69.2 \mu \mathrm{g} / \mathrm{mg} \pm 5.9 \mu \mathrm{g} / \mathrm{mg}$, respectively, $\mathrm{p}=0.23$ )

\section{DISCUSSION}

Despite the development of new urethral surgical techniques during recent decades, few studies on the structural arrangement of tissues used to correct this pathology are described. Until 
Figure 2 - Foreskin elastic system fibers. A) 28 years old smoker. Weigert x400. B) 28-years old non-smoker. Weigert $\mathbf{x} 400$. Note the marked increase of elastic fibers (brown) in the smokers compared to non-smokers. C) Immunohistochemistry with anti-elastin in the foreskin of $\mathbf{3 2}$ years old smoker patient showing elastic fibers (brown). Anti-elastin antibody X400. D) Immunohistochemistry with anti-elastin in the foreskin of $\mathbf{3 0}$ years old non-smoker showing fewer elastic fibers (brown). Anti-elastin antibody X400.
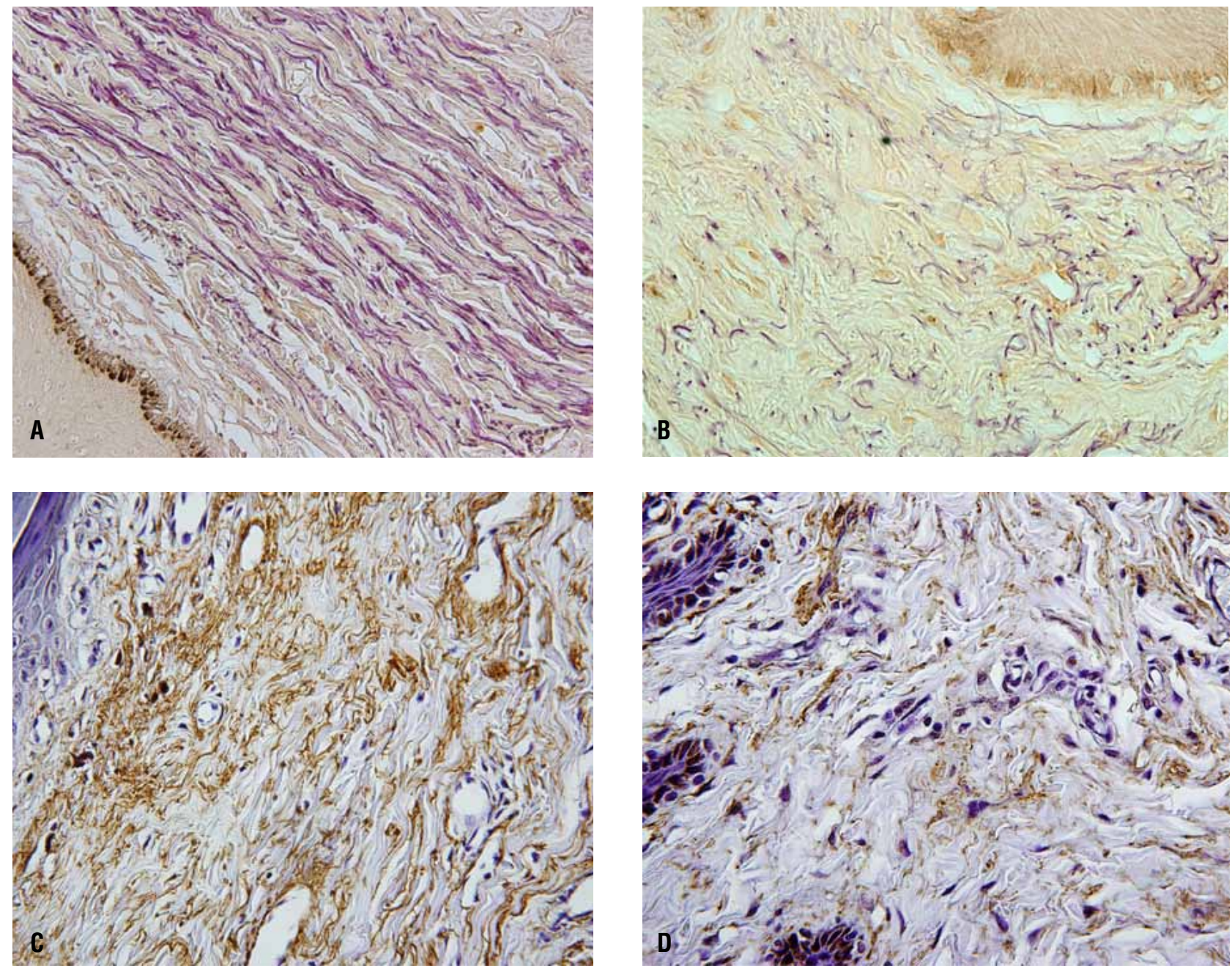

Table 1 - The table shows the results of stereological analysis of smooth muscle fibers and vascular density of the foreskin of the penis in smokers and the control group.

\begin{tabular}{|c|c|c|c|c|}
\hline & Smoker & Control & Smoker & Control \\
\hline Media & 13.0 & 12.46 & 9.44 & 10.17 \\
\hline SD & 1.00 & 1.88 & 0.97 & 1.22 \\
\hline$p$ value & \multicolumn{2}{|c|}{0.4297} & \multicolumn{2}{|c|}{0.1623} \\
\hline
\end{tabular}


now no study has focused on how smoking affects the structure of the foreskin employed as circular fasciocutaneous flaps to treat urethral stenosis. Whitson and McAninch (8-9) described that smokers experience worse results from tissue transfer urethroplasty, and Santucci (5) reported that the total impact of transverse distal penile island flap on the patient is still unknown, so further studies of postoperative complications are imperative. Harvesting the ventral based vascular dartos pedicle for urethral coverage either close to the skin on the ventral side or close to the penile corpus on the dorsal side are feasible techniques, with comparable success rates in terms of complications and cosmetic appearance (8-11). We agree with this affirmation since in this study we found no structural differences in the extracellular matrix between dorsal and ventral prepuce skin in smokers and non-smokers.

Several authors have reported the deleterious effects of smoking on healing. In a prospective observational study of 7224 plastic procedures, it was observed that smokers showed significantly more scar contour distortion demonstrating there are severe alterations in the wound healing caused by smoking, maybe due to structural alterations in the skin (12). Smokers experience significantly more frequent flap necrosis in breast reconstruction, regardless the type of reconstruction. This risk can increase up to 4.9 times $(13,14)$. Reus (15) studying patients with free skin grafts observed that smokers had significant ischemia $(p=0.03)$ at the graft edges.

In this study, we did not find any statistically significant difference in vascular density between smokers and non-smokers. Many authors have reported that the blood supply of the tissue at the reconstruction site is usually reduced by the most active component in tobacco, nicotine, which is known to cause vasoconstriction $(16,17)$, reducing the blood flow. This impairs wound healing and reduces oxygen delivery to the tissues. To assess the possible effects of smoking on the vascular functions in skin, all separate vascular lumens of the dermis were counted within five separate fields. The impossibility to differentiate microvasculature was a limitation of this study. Perhaps this made it impossible to detect altera- tions in vascular density of the foreskin. Another possibility would have been to measure the diameters of blood vessels, but this would have suffered the same problem of partial dimensional visibility.

Studies described that the synthesis of subcutaneous collagen in smokers is specifically impeded, indicating an impaired wound-healing process (18). According to Galatz (19) nicotine causes lower RNA expression for type I collagen and Tipton and Dabbous (20) found a higher concentration of collagenases. Nevertheless, there is no consensus on why the influence of nicotine reduces collagen synthesis. Furthermore, Machado (21), studying the healing of rabbit bladders, found no changes in collagen density in the scars. In the present study we found a reduced concentration of collagen of the foreskin in smokers, but without statistical significance.

Abnormalities in elastic fibers of smokers were reported by Francès (22) that found the elastic fibers in the skin of smokers were more numerous, wider and more fragmented than those of the skin of nonsmokers. The changes observed in elastic fibers look like those observed after solar damage, which affects the entire dermis, except that the papillary dermis in smokers remains unaffected. According to the author, the location of elastic damage caused by smoking may be related to the vascular distribution of the toxic substances in cigarette smoke. Boyd (23) compared the skin of 17 smokers and 14 non-smokers and reported increased elastosis in the sun-exposed skin of the forehead and cheeks of smokers compared to nonsmokers. He concluded that cigarette smoking is associated with an increase in elastosis, which may contribute to the clinical features of "smoker's face". That paper is important to confirm the alterations in elastic fibers in skin. More recently Just (24) reported that smoking is an independent risk factor for the increase of elastic fibers of the dermis of skin not exposed to the sun, and that acts on the two main structural components, elastin and microfibers.

Just as reported by those authors, we found 42.5\% more elastic fibers in smokers' foreskin tissue compared to that of the control group. Elastic fibers increase with age and this high concentration may partially explain the high extensibility 
of the skin in old-age. In vitro studies indicate that tobacco smoke extract impairs the production of collagen and increases the production of tropoelastin and matrix metalloproteinases (MMP), which degrade matrix proteins, and also causes abnormal production of elastosis material. Smoking increases MMP levels, which leads to the degradation of collagen and proteoglycans, suggesting an imbalance between biosynthesis and degradation in dermal connective tissue metabolism (25). Thus, in vitro and in vivo evidences indicate that smoking tobacco leads to accelerated aging of the skin, which probably explains the poor results in smokers where foreskin fasciocutaneous flaps are used in urethral reconstruction surgery.

Penile circular fasciocutaneous flap necrosis is significantly more frequent in smokers, probably due to foreskin structural alterations as described above. We warn that urethral reconstruction surgery using penile circular fasciocutaneous flaps should be done with caution in smokers and the patient should be strongly advised to stop smoking before surgery. The adverse effects of smoking on the appearance and function of the skin could also be used in health education, to discourage people from starting to smoke and to encourage current smokers to quit.

\section{CONCLUSION}

The foreskin tissue of smokers had significantly more elastic fibers. Elastic fibers play an important role in this tissue turnover and this high concentration in smokers possibly causes high extensibility of the foreskin. The structural alterations in smokers' foreskin could possibly explain the poor results in such patients submitted to foreskin fasciocutaneous flaps in urethral reconstruction surgery.

Supported by grants from the National Council of Scientific and Technological Development (CNPQ - Brazil) and Fundação Carlos Chagas Filho de Amparo à Pesquisa do Estado do Rio de Janeiro (FAPERJ), Brazil.

\section{CONFLICT OF INTEREST}

None declared.

\section{REFERENCES}

1. Olajide AO, Salako AA, Aremu AA, Eziyi AK, Olajide FO, Banjo 00: Complications of transverse distal penile island flap: urethroplasty of complex anterior urethral stricture. Urol J. 2010; 7: 178-82.

2. Cavalcanti AG, Costa WS, Baskin LS, McAninch JA, Sampaio FJ: A morphometric analysis of bulbar urethral strictures. BJU Int. 2007; 100: 397-402.

3. Fenton AS, Morey AF, Aviles R, Garcia CR: Anterior urethral strictures: etiology and characteristics. Urology. 2005; 65: 1055-8.

4. Santucci RA, Joyce GF, Wise M: Male urethral stricture disease. J Urol. 2007; 177: 1667-74.

5. Santucci RA, Mario LA, McAninch JW: Anastomotic urethroplasty for bulbar urethral stricture: analysis of 168 patients. J Urol. 2002; 167: 1715-9.

6. Barbagli G, Palminteri E, Guazzoni G, Montorsi F, Turini D, Lazzeri M: Bulbar urethroplasty using buccal mucosa grafts placed on the ventral, dorsal or lateral surface of the urethra: are results affected by the surgical technique? J Urol. 2005; 174: 955-7; discussion 957-8.

7. Wessells H, McAninch JW: Current controversies in anterior urethral stricture repair: free-graft versus pedicled skin-flap reconstruction. World J Urol. 1998; 16: 175-80.

8. Whitson JM, McAninch JW, Elliott SP, Alsikafi NF: Long-term efficacy of distal penile circular fasciocutaneous flaps for single stage reconstruction of complex anterior urethral stricture disease. J Urol. 2008; 179: 2259-64.

9. Breyer BN, McAninch JW, Whitson JM, Eisenberg ML, Mehdizadeh JF, Myers JB, et al.: Multivariate analysis of risk factors for long-term urethroplasty outcome. J Urol. 2010; 183: 613-7.

10. Cabral CA, Sampaio FJ, Cardoso LE: Analysis of the modifications in the composition of bladder glycosaminoglycan and collagen as a consequence of changes in sex hormones associated with puberty or oophorectomy in female rats. J Urol. 2003; 170: 2512-6.

11. Bergman I, Loxley R: The determination of hydroxyproline in urine hydrolysates. Clin Chim Acta. 1970; 27: 347-9.

12. Dixon AJ, Dixon MP, Dixon JB, Del Mar CB: Prospective study of skin surgery in smokers vs. nonsmokers. Br J Dermatol. 2009; 160: 365-7.

13. Al-Qudah HS, Santucci RA: Extended complications of urethroplasty. Int Braz J Urol. 2005; 31: 315-23; discussion 324-5.

14. Burgu B, Aydogdu O, Söylemez H, Soygur T: Both dorsal and ventral flaps can be used in previously circumcised hypospadic adults with comparable success rates. Int Urol Nephrol. 2010; 42: 689-95.

15. Reus WF 3rd, Colen LB, Straker DJ: Tobacco smoking and complications in elective microsurgery. Plast Reconstr Surg. 1992; 89: 490-4. 
16. Padubidri AN, Yetman R, Browne E, Lucas A, Papay F, Larive $B$, et al.: Complications of postmastectomy breast reconstructions in smokers, ex-smokers, and nonsmokers. Plast Reconstr Surg. 2001; 107: 342-9; discussion 350-1.

17. Goodwin SJ, McCarthy CM, Pusic AL, Bui D, Howard M, Disa $\mathrm{JJ}$, et al.: Complications in smokers after postmastectomy tissue expander/implant breast reconstruction. Ann Plast Surg. 2005; 55: 16-19; discussion 19-20.

18. Haustein KO: Smoking tobacco, microcirculatory changes and the role of nicotine. Int J Clin Pharmacol Ther. 1999; 37: 76-85.

19. Galatz LM, Silva MJ, Rothermich SY, Zaegel MA, Havlioglu $\mathrm{N}$, Thomopoulos S: Nicotine delays tendon-to-bone healing in a rat shoulder model. J Bone Joint Surg Am. 2006; 88: 2027-34.

20. Tipton DA, Dabbous MK: Effects of nicotine on proliferation and extracellular matrix production of human gingival fibroblasts in vitro. J Periodontol. 1995; 66: 1056-64.

21. Machado C, Chin EW, Ioshii So, Tâmbara Filho R, Von Bathen A: Influence of nicotine on healing of vesical sutures in rabbits. Acta Cir Bras. 2007; 22: 479-84.
22. Francès $C$, Boisnic $S$, Hartmann DJ, Dautzenberg $B$, Branchet MC, Charpentier YL, et al.: Changes in the elastic tissue of the non-sun-exposed skin of cigarette smokers. Br J Dermatol. 1991; 125: 43-7.

23. Boyd AS, Stasko T, King LE Jr, Cameron GS, Pearse AD, Gaskell SA: Cigarette smoking-associated elastotic changes in the skin. J Am Acad Dermatol. 1999; 41: 23-6.

24. Just M, Ribera M, Monsó E, Lorenzo JC, Ferrándiz C: Effect of smoking on skin elastic fibres: morphometric and immunohistochemical analysis. Br J Dermatol. 2007; 156: 85-91.

25. Morita A: Tobacco smoke causes premature skin aging. J Dermatol Sci. 2007; 48: 169-75.

\section{Correspondence address:}

Dr. Luciano Alves Favorito Rua Professor Gabizo 104 / 201 20271-062, Rio de Janeiro, RJ, Brazil Fax: + 5521 3872-8802

E-mail: lufavorito@yahoo.com.br 